\title{
Correlation between halo coronal mass ejections and solar surface activity ${ }^{\star}$
}

\author{
Guiping Zhou ${ }^{1,2}$, Jingxiu Wang ${ }^{1}$, and Zhuoliang $\mathrm{Cao}^{2}$ \\ ${ }^{1}$ National Astronomical Observatories, Chinese Academy of Sciences, Beijing 100012, PR China \\ e-mail:wjx@ourstar.bao.ac.cn; zhougp@ourstar.bao.ac.cn \\ 2 Department of Physics, AnHui University, HeFei 230039, PR China \\ e-mail: caoju@mars . ahu.edu.cn
}

Received 22 May 2002 / Accepted 17 September 2002

\begin{abstract}
We survey all the coronal mass ejections (CMEs) observed by the Large Angle and Spectrometric Coronagraph Experiment (LASCO) aboard the Solar \& Heliospheric Observatory (SOHO) in the interval from 1997 to 2001, and select 197 frontside halo CMEs whose associated near-surface activity could be clearly identified from SOHO EUV Imaging Telescope (EIT) and other space-borne and ground-based observations. A statistical analysis has been made with the emphasis on the correlation between CMEs and solar surface activity.

We have found in our sample that all the CMEs were accompanied by local brightening in the CME source regions in EIT and/or $\mathrm{H} \alpha$ images. However, if we only classify the events with a brightening increase more than $50 \%$ above the quiet background as flares, as measured from EIT data, approximately $88 \%$ of the earth-directed CMEs are associated with flares and more than $94 \%$ are associated with eruptive filaments. With regard to the locations of CME source regions, there are about $79 \%$ CMEs initiating from active regions, while only $21 \%$ originate outside active regions. We evaluate the symmetry between CMEs and associated solar surface activity in spatial distributions. We find that in about half the events the surface activity is asymmetric with respect to the CME. For the flares having GOES X-ray records in this study, allowing for the uncertainties specified by the data cadence, $59 \%$ of the CME initiations seem to precede the flare onset in the CME source regions, while $41 \%$ are preceded by flare onsets. The statistical correlation seems to suggest that CMEs are intrinsically related to surface activity.
\end{abstract}

Key words. Sun: coronal mass ejections (CMEs) - Sun: flares - Sun: filaments

\section{Introduction}

CMEs are the most spectacular form of solar activity. They often consist of very large structures containing plasma and magnetic fields that are expelled from the Sun into the heliosphere. Most of the ejected material comes from the low corona, although cooler, denser material, probably of chromospheric origin, can also be ejected (Webb 2000). Compared with solar flares and filaments, CMEs are relatively new to astronomers. In fact, their analogues have just started to be identified in other astrophysical subjects (Bond et al. 2001). Since CMEs were first detected in the early 1970 , the solar community has tried to understand whether or not this newly discovered phenomenon is intrinsically related to the other well-known solar activities, such as flares, filament eruptions and specific types of radio bursts. Both case studies and statistical approaches have been

Send offprint requests to: G. p. Zhou, e-mail: zhougp@ourstar.bao.ac.cn

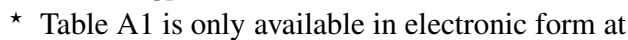
http://www. edpsciences.org made in this direction. The study of the association of CMEs with other forms of solar activity has a multi-fold importance. Firstly, such association will help us to understand the physical links between the very large-scale activity (e.g. CME) and the rather small-scale phenomena (e.g. flares and filament eruptions). Secondly, we need to understand the common physics behind the varied forms of solar activity, to learn how the difference in spatial scale specifies the physical properties. Thirdly, in the narrow sense of CME studies, without knowledge of the associated surface activity, the CME initiation will not be properly understood.

Before the SOHO mission, Munro et al. (1979) carried out the first detailed surveys associating about 75 major Skylab CMEs viewed in the middle corona with near-surface activity from 1973-1974, in combination with reports of associated activity from the Solar Geophysical Data (SGD) archives. They found that $75 \%$ of the CMEs observed were associated with other forms of solar activity, $40 \%$ of the CMEs observed were associated with flares, $50 \%$ with eruptive prominences solely (without flares), and more than $70 \%$ of CMEs with eruptive 
prominences (with and without flares). Webb \& Hundhausen (1987) examined Solar Maximum Mission (SMM) data during the activity maximum in 1980 and found that about two thirds of the CMEs observed were associated with other forms of solar activity. Among them, $68 \%$ of the observed CMEs were found to be associated with erupting prominences, $37 \%$ with $\mathrm{H} \alpha$ flares, and 47\% with X-ray events. Cyr \& Webb (1991) performed similar studies with SMM data from 1984 to 1986. Slightly less than half of the CMEs were found to have associations with other forms of solar activity. The results were that $76 \%$ were associated with erupting prominences, $26 \%$ with $\mathrm{H} \alpha$ flares, and $74 \%$ with X-ray events.

After the SOHO mission, the LASCO coronagraphs and the EUV Imaging Telescope (EIT) aboard SOHO provided the opportunity for CME studies. SOHO allows for solar observations uninterrupted by a day-night cycle. Yohkoh SXT has a higher time cadence and continuity of operation than previous space-borne X-ray telescopes. The combined observations from LASCO with SXT and EIT for imaging the rather hotter and cooler corona, respectively, give us unprecedented opportunities to study CME coronal sources (Sterling 2000). Hudson et al. (1998) and Webb et al. (2000) both examined halo-type CMEs and their associated solar activity observed during the first half of 1997, and found that about half of the halo events were associated with frontside activity, as expected. Gilbert et al. (2000) analyzed $54 \mathrm{H} \alpha$ events from February 1996 to June 1998 to study the relationship between prominence activity and CMEs. They found that $94 \%$ of eruptive prominences (EPs) had an associated CME and $76 \%$ of the CMEs were associated with eruptive prominences in their study. Subramanian \& Dere (2001) examined 32 CMEs from January 1996 to May 1998. They also investigated the relationship between CMEs and prominence eruptions, and found that $44 \%$ of the CME-related transients were associated with eruptions of prominences embedded in active regions, and $15 \%$ with eruptions of prominences outside active regions.

There are also some case studies of the association of CMEs and surface activity. Zhang et al. (2001a) studied the temporal relationship between coronal mass ejections and flares. They concluded that the impulsive acceleration phase of CMEs coincided well with the rise phase of the accompanying $\mathrm{X}$-ray flares. While the duration of the rise phase of flares can be a few minutes to tens of minutes, the corresponding CMEs always showed an apparent speed increment along with that of the soft X-ray flux. Recently, Gopalswamy et al. (1999) reported possibly the first observation of a large-scale X-ray enhancement, not dimming, associated with a filament eruption that could be the X-ray counterpart of a CME. Harrison et al. (2000) made a spectroscopic study of coronal dimming associated with a CME. They concluded that the dimming was consistent with the loss of significant mass, which could account for at least $70 \%$ of the mass of the CME. It was stressed that the dimming event was most likely the source region of at least a large fraction of associated CME mass.

Though so many studies have been performed, there are still many suppositions about the correlation between CMEs and surface activity. Since the famous paper "The solar flare myth" was published by Gosling (1993), it would be prudent to search for correlations and avoid sweeping and unwarranted conclusions.

Halo CMEs are those that originate from the disk and appear as expanding, circular brightening that completely surrounds or spans a large angle outside the coronagraph occulter, which blocks the bright solar photospheric light. The observations suggest that these CMEs are spherically symmetric structures moving outward either toward or away from Earth that are detected after expanding to a size exceeding the diameter of the coronagraph's occulter (Webb 2000). The first halo CME was reported by Howard et al. (1982), using observations made with the Solwind coronagraph on the P78-1 spacecraft. Several halo-CME events have been reported by LASCO (Plunkett et al. 1998; Thompson et al. 1998). Halo CMEs are particularly useful for studying CME coronal sources, since most of their source regions are expected to be near the center of the solar disk for those directed toward the Earth (Sterling 2000). The study of halo CMEs would help us to establish a correlation between disk active phenomena and CMEs observed outside the solar limb, to understand their initiation mechanism and, eventually, to forecast when the eruptions will occur (Zhang et al. 2001b). Before the SOHO mission, halo-type CMEs had been rarely reported in coronagraph observations of the Sun (e.g. Howard et al. 1982). However, with the increased field of view and sensitivity of LASCO, such CMEs have been detected at a rate of $1-3$ per month, or about $11 \%$ the rate of all LASCO CMEs (Cyr et al. 2000; Webb 2000). To date, LASCO has observed from solar minimum well into the maximum phase of the current (the 23rd) solar cycle and has detected CMEs at a rate slightly higher than but comparable to the earlier observations (Howard et al. 1997; Cyr et al. 1997, 2000; Webb 2000).

Thanks to the great success of SOHO and Yohkoh, the disk observations of CME initiation become possible, particularily for the earth-directed halo CMEs. To avoid the ambiguity in locating CME source regions, as argued by Feyman \& Martin (1995), only frontside halo CMEs are selected in this statistical study. A CME with span angle greater than $130^{\circ}$ is referred to as a halo CME in our approach. There were 519 halo CMEs identified from 1997 to 2001, an interval roughly covering the Solar Minimum to Maximum in this solar cycle. The statistics are made with the emphasis on the correlation between CMEs and surface activity in terms of flares and filament eruptions.

In the next section, we introduce the data base and event selection. In Sect. 3, we demonstrate the categories and examples of CME associated surface activity. In Sect. 4, we present our statistical results. Section 5 gives concluding remarks. Finally, we place a table about all the sampling CMEs and associated surface activity in an appendix.

\section{Data analysis}

\subsection{Data base}

The primary data bases for this study are SOHO LASCO and EIT time-lapse observations. The advantage of the data base is the complete temporal coverage of the observations. However, both LASCO and EIT have rather poor temporal resolution. To clearly identify the earth-directed halo CMEs and their 
associated surface activity, other ground-based and spaceborne observations are necessary. Among them, the GOES Xray counts, Big Bear $\mathrm{H} \alpha$ filtergrams, Yohkoh SXT images are very useful to identify the CME source regions, as well as flares and filament eruptions.

In the study, we also use observations from Huairou Solar Observing Station (HSOS), Hiraiso Solar Terrestral Research Center (HSTRC), Holloman Air Force Base (HAFB) and others. To learn whether or not the CME source regions are located inside active regions, SOHO MDI daily magnetograms and synoptic charts are also referred to.

\subsection{Sampling the frontside CMEs}

Since there were few high-cadence EIT $195 \AA$ observations before March 1997 because of telemetry restrictions (Subramanian \& Dere 2001), we select our data after March 1997. Firstly, we obtain all the halo CMEs whose angular widths are greater than $130^{\circ}$ and then record the approximate time, TM, when they initiated from the solar disk according to the linear fit of height-time from SOHO LASCO CME Category (see http://cdaw.gsfc.nasa.gov/CME_list/). At the same time, we record the CME first appearance time in $\mathrm{C} 2$ and their directions in $\mathrm{C} 2 / \mathrm{C} 3$. We find 519 such halo CMEs.

Secondly, we examine corresponding EIT movies and EIT running difference (RD) movies to check whether these halo CMEs have counterparts on the visible solar disk. Here two criteria are used to identify frontside halo CMEs. The first criterion is that the surface activity starts in the time window $\mathrm{TM} \pm 30 \mathrm{~min}$ (Wang et al. 2002). The surface activity includes filament eruptions, flaring, dimming, plasma ejecta and so on. We also grid over EIT images to locate the position of solar surface activity termed as PSSA. The second criterion is that the PSSA is under the span of the associating CME. In order to determine the span of the CMEs, the position angle (PA) of the two outer edges of the CME were measured counterclockwise from Solar North in degrees on the sky plane. The PA measurements were generally made using $\mathrm{C} 2$ data. If $\mathrm{C} 2$ data were not available, $\mathrm{C} 3$ data were used. In the case that the apparent width of a CME gradually increased with time, the PA was measured when the width reached maximum. The difference between the two edge PAs, i.e. the apparent angle width fanned out by the CME, determined the CME span (Fig. 1A), which was measured by Yashiro et al. of the SOHO team, and is presented at http://cdaw.gsfc.nasa.gov/CME_list/ (Yashiro et al. 2002). Measured counterclockwise, the angle between the line along the Solar North and the line connecting the center of CME source region and the solar disc center defines the source region position angle (SRPA) (Fig. 1B) for each selected CME. If a CME's SRPA is within the range of the CME's span, the second criterion is satisfied. Since CME front loops were rather clear in $\mathrm{C} 2 / \mathrm{C} 3$ images, it is very easy to identify whether the second criterion is satisfied. For a few events, their associated surface activity positions were just outside or on the edge of the CME spans. However, from the EIT and EIT RD movies, we can clearly identify the propagation directions of EIT dimming or plasma ejecta that coincided with that of the CMEs.
Those surface events are also clarified as being under the spans of the given CMEs. If a CME is associated with such surface activity which satisfies the above two criteria, we identify the CME as frontside one.

During the process, we cut off several data gaps in which there are no records in the SOHO LASCO CME Category, such as July-September 1998 and January 1999, or no EIT data or high-cadence EIT data. As a result, 197 frontside halo CMEs are definitively identified and selected in this study. They are listed in Table A1 of the Appendix

\subsection{Grouping the surface activity}

In this study, we only take two primary forms of solar activity, the flares and filament eruptions, into account. Some other active manifestations, e.g. EIT wave and/or dimming, are considered as secondary active phenomena of activity.

To identify associated flares, we first scrutinize timelapse images. After a candidate is selected, we search its corresponding X-ray records from the GOES satellite in the Space Physics Interactive Data Resource (SPIDR, see http://spidr.ngdc.noaa.gov/). We compare the starting time and positions of the flares in EIT $195 \AA$ Amages with those of X-ray flares recorded in SPIDR. If a flare seen in EIT images takes place within the duration of a X-ray burst and their position difference is within the range of $\pm 5^{\circ}$ in both the latitude and longitude, we regard the two sets of observations as the same event. Because of low-cadence EIT observations and the different methods of measuring flare positions, generally, such errors are tolerable. Then we record the class, startingmaximizing-ending time and duration of the associated flares in SPIDR. Among 197 halo CME-associated solar surface events, 141 events have been classified as flares with GOES X-ray records. Since soft $\mathrm{X}$ rays and $\mathrm{H} \alpha$ wavebands each contain only $5-10 \%$ of the total radiated energy, it may be not proper to identify a flare with only its soft X-ray light curve, as recorded by GOES (Hudson 1995). With regard to the events not listed in $\mathrm{X}$-ray flare categories, we have measured the increased intensity during the activity. We define the intensity increase as the ratio of brightness increase divided by the background intensity of the same prepared EIT image in a little disturbed area. If the percentage is greater than $50 \%$, we classify it as a flare, otherwise this event is regarded as an activity event without flare.

Compared with flares, CME-associated erupting filaments are more difficult to confirm since not all the filaments have available $\mathrm{H} \alpha$ data in our sample. To ascertain filament eruptions solely from EIT observations is not easy. We adopt two criteria in identifying a filament eruption. They are: a) dark and/or bright plasma ejecta, which is an empirical criterion proposed by Subramanian \& Dere (2001); b) appearance of two flaring ribbons and post-flare loops, which is suggested by this paper. If either or both of the two above manifestations can be identified in EIT movies, we would classify the event as having filament eruptions. Considering the limitation of our available data base and the clearness in physics, we only group the solar surface activity into the following three categories:

A(a)-Flares with obvious filament eruptions; 

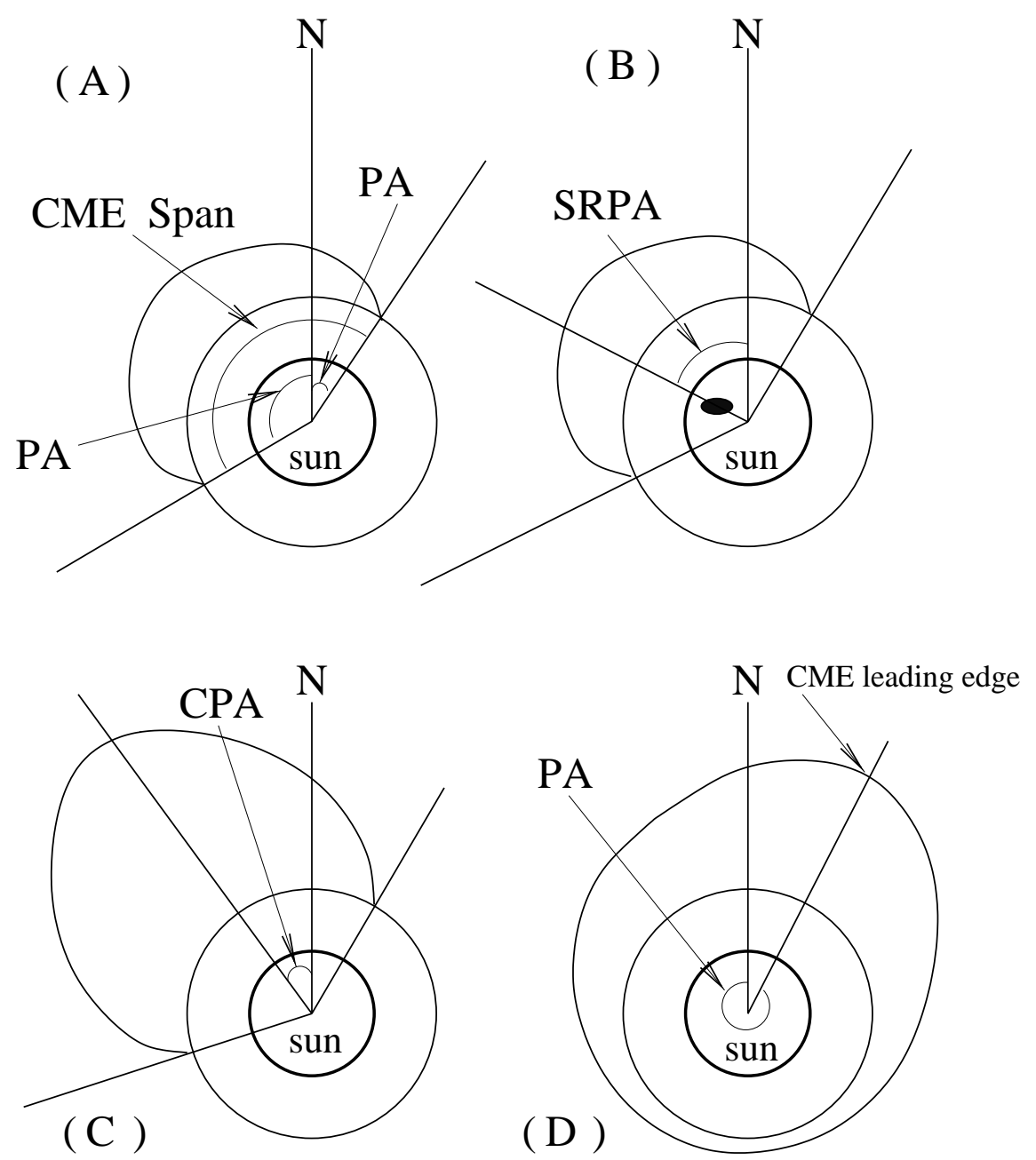

Fig. 1. Definitions of the span, SRPA, CPA and PA of a CME.

A(b)-Flares which may have been preceded by filament eruptions but cannot be confirmed by the available data base;

B-Filament eruptions with too little brightening to be termed flares.

Now, we evaluate the symmetry between CMEs and associated solar surface activity in spatial distributions. Here there are two types of angles to be applied. One is the central position angle (CPA), which is in accordance with the CMEs whose spans were less than $360^{\circ}$. The CPA was defined as the the mid angle with respect to the two edges of the CME in the sky plane (Fig. 1C). The other is the PA, an angle between the line along the Solar North and the line connecting the CME's fastest leading edge and the solar disc center (see Fig. 1D). This is in connection with the halo CMEs whose spans were $360^{\circ}$. Such PA measurements were made in each frame where the CME's leading edge can be identified above the noise level. These PAs and CPAs were measured by Yashiro et al. and are found at http: //cdaw.gsfc .nasa.gov/CME_list/ (Yashiro et al. 2002). Also, as there are some uncertainties in SRPA determinations, an uncertainty of 10 degrees is estimated. If the difference between SRPA and CPA or PA (to $360^{\circ}$ halo CMEs) of a $\mathrm{CME}$ is not greater than $20^{\circ}$, we define the event as symmetric, or else it is asymmetric.

\section{Examples}

Here we select three well-observed events from the list of 197 events as examples to demonstrate how we identify and classify these events. They are listed in Table 1.

\subsection{January 20 CME}

This is an example of category A(a), CMEs associated with flares and clearly identified filament eruptions.

At first, the CME appeared in $\mathrm{C} 2$ as a bright patch in the east at 19:32 UT, marked by the arrow in Fig. 2A. Its bright leading edge of the halo was starting over the east limb and later had $360^{\circ}$ emission around the disk. According to the heighttime profile curves which were back-extrapolated by LASCO groups, we know that the onset time of the CME was near 18:36 UT. The wide front on the East limb travelled outward at an average linear speed of $839 \mathrm{~km} \mathrm{~s}^{-1}$. In general, there would be errors of about 30 min between the extrapolation time and the real starting time when the CME initiated from the solar disk. We consider this error in judging the associated solar surface activity. There was clear extension of this patch above the north and south pole, and apparently extended to surround the full disk later. It is possible that the activity happened in 
Table 1. Examples.

\begin{tabular}{lllll}
\hline \hline Time & Category & NOAA & X-ray class & Active region \\
\hline 2001 January 20 & A(a) & 9313 & M1.2 & Inside \\
2001 April 06 & A(b) & 9415 & X5.6 & Inside \\
2000 May 08 & B & No & No & Outside \\
\hline
\end{tabular}

the east hemisphere. This halo CME showed maximum intensity and extension in the east limb.

Figure $2 \mathrm{~B}$ is a $\mathrm{BBSO} \mathrm{H} \alpha$ image. In this image, a filament lay in the AR9313 at 08:04 UT before the initial CME time. Figure 2C is a MDI image that shows AR9313, where the activity happened.

More detailed information about solar disk magnetic activity is provided by EIT $195 \AA$ images. The right column of Fig. 2 is a time sequence of EIT $195 \AA$ images which show the typical characteristics of Category A(a). From the corresponding EIT movie, we can observe both the bright and dark ejecta (showed in panel D) which indicate filament eruptions. There was bright EIT wave propagating eastward. It was accompanied by a M1.2 flare that started at 18:33 UT, peaked at 18:47 UT and ended at 18:59 UT. The initial time of CME was at 18:36 UT which was close to the flare onset time. Its direction coincided with that of the propagating dimming. Figures $2 \mathrm{E}$ and $2 \mathrm{~F}$ show the typical two ribbons and post-flare loops of the eruptive flare (see arrows in Figs. 2E and 2F). This was an eruptive flare.

In some cases, since there are no available $\mathrm{H} \alpha$ data, and no obvious ejecta could be seen in the rather low-cadence EIT $195 \AA$ images; The signatures with two ribbons and post-flare loops are regarded as another criterion of filament eruptions. Meanwhile, EIT $195 \AA$ RD images can suggest that the propagating depletion initiated from the active region which accompanied by material ejection spreading preferentially toward a certain direction. This also can help us to identify the associated filament eruptions.

Since this CME angular width was $360^{\circ}$, its PA measured as $64^{\circ}$ was regarded as the symmetric axis. Its associated solar surface activity was located in S07E40 where the SRPA was $100^{\circ}$. It is obvious that the position angle difference between the CME and associated solar surface activity was greater than $20^{\circ}$. Thus, the surface activity was asymmetric in spatial position with regard to the CME span.

\subsection{CME of April 6, 2001}

Figure 3 shows a CME example on April 6, 2001 off the southeast solar limb. As seen in the $\mathrm{C} 2$ coronagraph, the bright loop fronts of the CME filled the southeast quadrant. Its full halo in C2 appeared at 20:06 UT and was faint to the west and much fainter to the north. Unless otherwise stated, this is a RD LASCO image. The first appearance of the CME in C2 was at 19:30 UT. Extrapolation of the height-time plots back to $1 R_{\odot}$ showed the CME leaving at 18:54 UT, $36 \mathrm{~min}$ before the first CME appearance. The average linear velocity was $1270 \mathrm{~km} \mathrm{~s}^{-1}$. This was a fast CME.
Figure $3 \mathrm{C}$ is a $\mathrm{H} \alpha$ image which shows a filament in the AR 9415. The source region of this CME was in the rather compact AR9415 (Fig. 3B). The EIT $195 \AA$ movie showed the development of the X5.6 flare in AR9415 and the northeast streamer. For simplicity, we only use an EIT image and an EIT RD image (Figs. 3D and 3E) to show the flare and the propagating dimming. This flare started at 19:10 UT, peaked at 19:21 UT and ended at 19:31 UT, while the CME initiation was at 18:54 UT, before the flare onset. Because the cadence of EIT images (approximately $7-15 \mathrm{~min}$ ) is generally poor and leads to miss a large fraction of short-lived, weak, transient events, a cadence faster than one image per minute is probably required (Biesecker \& Thompson 2000). We are uncertain whether this filament erupted to trigger the flare or not since the characteristics of the filament eruption in the EIT $195 \AA$ images are not obvious. Figure $3 \mathrm{E}$ is a RD image of EIT $195 \AA$ A showing the dimming. The dimming mainly resulted from the flare, as we find that there were propagating dark areas in phase with the flaring, seen in dark in the RD EIT movie. The dimming appeared to be one of the earliest and best-defined signatures in soft $\mathrm{X}$-ray and EUV emission of the mass ejection in the low corona (Webb 2000). However, we cannot ascertain whether the filament erupted to cause such mass ejections. We notice that some dimming areas appeared close to the Sun limb, shown by the arrows in Fig. 3E, and propagated first to the southeast, then passed the disk through active regions, which were associated with a CME observed by LASCO C2 (see Fig. 3A).

This was also a halo CME with $360^{\circ}$ angular width. Its position angle was $147^{\circ}$. The associated solar surface activity was located in S19W53 where the SRPA was about $115^{\circ}$. The difference between the two position angles was greater than $20^{\circ}$. The event is regarded as an asymmetric one.

This CME associated with a flare and its source region has a filament in corresponding $\mathrm{H} \alpha$ images, but the characteristics of the filament eruption are not obviously observed. We place it in Category $\mathrm{A}(\mathrm{b})$. It is likely that there would be filament eruptions in most $\mathrm{A}(\mathrm{b})$ events.

\subsection{CME of May 8,2000}

The CME of May 8, 2000 was a slower one compared with the two above events. Its front loops were very weak in LASCO $\mathrm{C} 2$ and were relatively clear in $\mathrm{C} 3$ as shown in Fig. 4A. By 14:26 UT, the edge of the CME moved into the C2 field of view and appeared in the southwest. The linear fit speed of the CME was $465 \mathrm{~km} \mathrm{~s}^{-1}$ for the leading edge of the CME. Backextrapolations of the CME's counterparts on the solar disk happened around $1 R_{\odot}$ at 12:48 UT. 


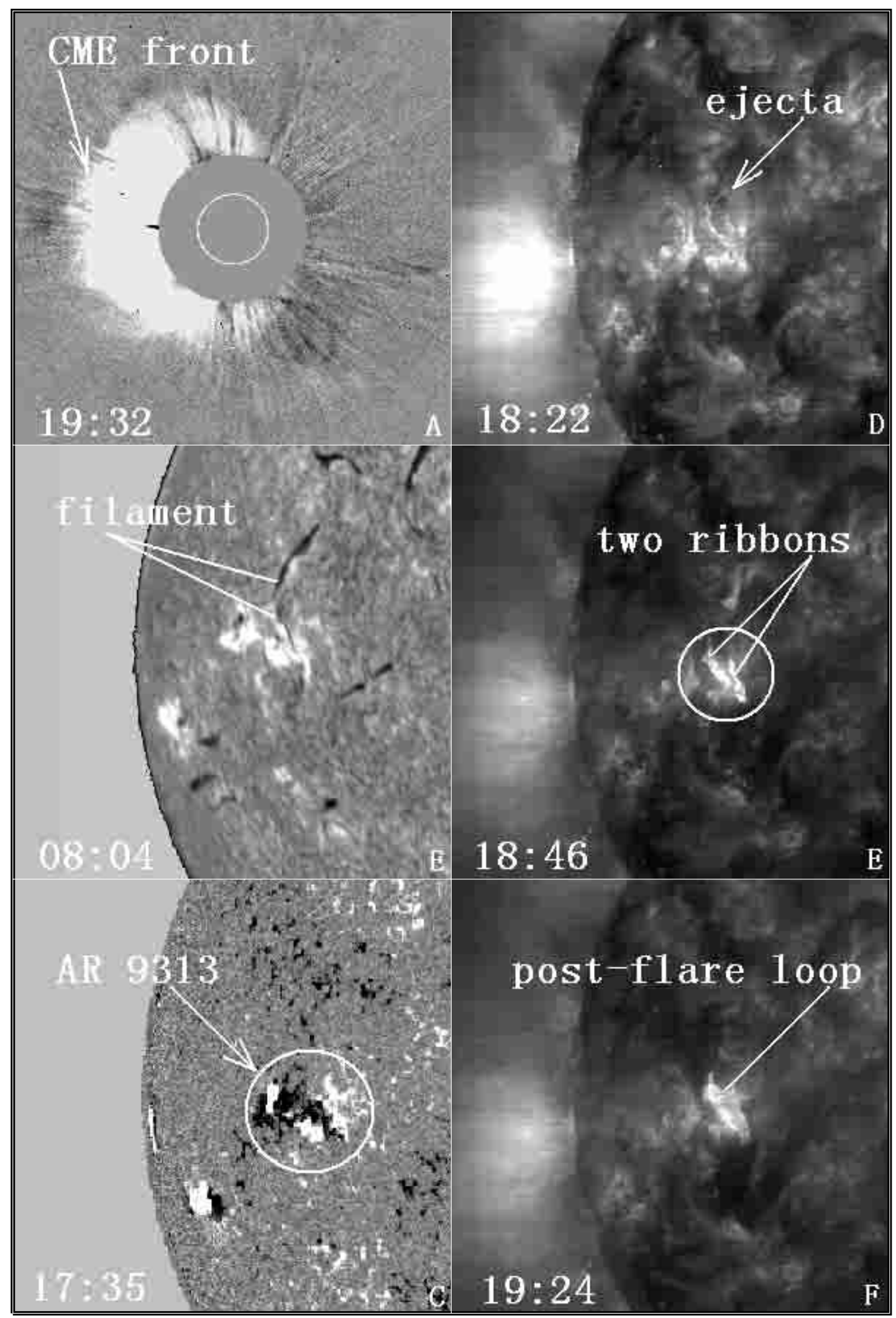

Fig. 2. A) An RD image of the halo CME obtained by LASCO/C2 on January 20, 2001. Its bright front firstly appeared toward the south. B) The H $\alpha$ image at 08:04 UT. C) The MDI image at 17:35 UT. D), E) and F) are EIT $195 \AA$ A images which show the development of the solar surface activity.

Figure $4 \mathrm{~B}$ is an $\mathrm{H} \alpha$ image from the BBSO. A filament is visible in a plage region. We present the MDI magnetogram at 12:48 UT to show that the source region was in a plage region (Fig. 4C). This activity happened in S29W14. The right 


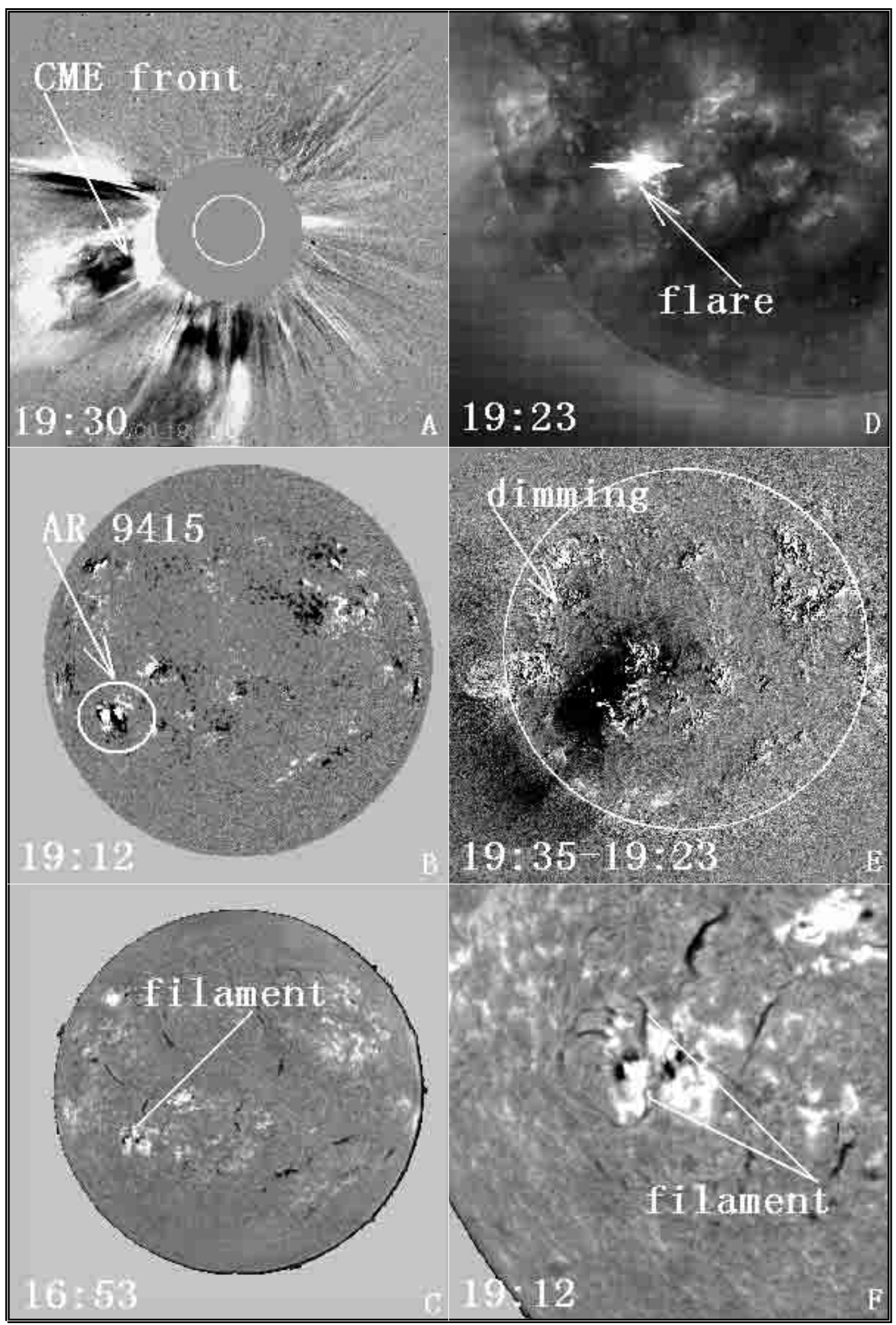

Fig. 3. A) An RD image of the halo CME obtained by LASCO/C2 on April 6, 2001. B) The MDI image at 19:12 UT. C) A filament seen in the AR 9415 in the $\mathrm{H} \alpha$ image at 16:53 UT from BBSO. D) An EIT $195 \AA$ image that show the X5.6 flare. E) An EIT RD image indicates the propagating dimming. F) A part of the enlarged image D) which clearly shows the filament in AR9415.

panels are a series of EIT images on May 8, 2000 which show the development of the filament eruption indicated by white arrows. We have examined all the EIT images from 12:00 UT to 17:00 UT. There was no corresponding GOES X-ray flare 


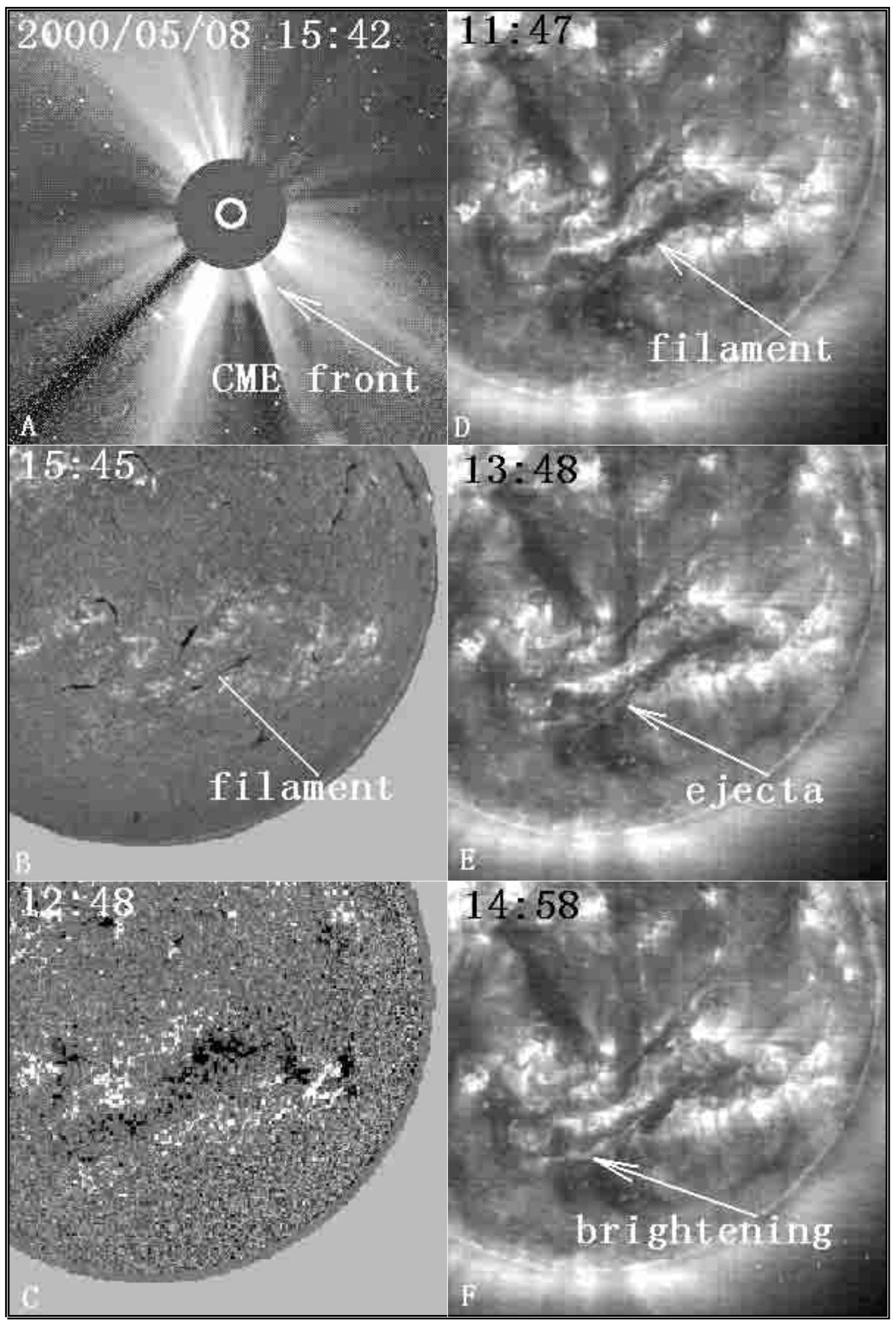

Fig. 4. A) An image of the halo CME obtained by LASCO/C3 on May 8, 2000. B) The H $\alpha$ image at 15:45 UT. C) The MDI image at 12:48 UT. D), E) and F) are EIT $195 \AA$ Amages that show the development of the erupting filament.

record in this region, but there was a weak brightening accompanying by the filament eruption. The measured intensity increase is $7 \%$, which is not high enough to be classified as a flare. We regard the CME as one which was only associated with filament eruption without flare. However, it is worth emphasizing that if the intensity increase is not small compared 
Table 2. Categories of associated solar surface activity.

\begin{tabular}{llll}
\hline \hline Category & Associated Surface Activity & Number & Percentage (\%) \\
\hline A(a) & flares and filament eruptions & 162 & 82.2 \\
A(b) & flares and possible filament eruptions & 11 & 5.6 \\
B & filament eruptions with little brightening & 24 & 12.2 \\
\hline Total & & 197 & \\
\hline
\end{tabular}

with that of the quiet background, the filament eruption and the following brightening have almost the same characteristics as those of an eruptive flare.

The angular width of this $\mathrm{CME}$ was $137^{\circ}$ which was less than $360^{\circ}$, so the CPA was selected as the symmetric axis. Its CPA was $216^{\circ}$, while its associated solar surface activity was located in S29W14 where the SRPA was $210^{\circ}$. Obviously, it was an symmetric event.

\section{Statistical results}

We use the observations from LASCO, EIT, GOES X-ray, $\mathrm{H} \alpha$ and synoptic and daily MDI magnetograms to examine the relationship between halo CMEs and the solar surface activity during 1997 March-2001 December. We have recorded the heliographic coordinate of the CME associated solar surface activity in $195 \AA$ EIT data for each event in our sample. In this study, we find that almost all the CMEs are accompanied by local brightening in the CME source regions. Most of these events except for 32 events in Category $\mathrm{A}(\mathrm{a})$ and $\mathrm{A}(\mathrm{b})$ have flare records in GOES X ray. Those 32 events whose intensity increase are greater than $50 \%$, as defined in Sect. 2.3, were also regarded as flare events. Judging filament eruptions only from EIT $195 \AA$ images is not adequate. We have employed a variety of source data to ascertain whether a CME is associated with an erupting filament. $\mathrm{H} \alpha$ data from the BBSO, and some other sources like the HAFB and the HSOS, are used to find out whether a filament existed near or in the source region of the CME-related transient. But not all the events have available $\mathrm{H} \alpha$ data. Since eruptive flares suggest that there are often filament eruptions triggering flares, we adopt two further criteria to judge filament eruptions from EIT $195 \AA$ data. The association of CMEs with surface activity is summarized in Table 2.

All the sampling CMEs are associated with solar surface activity. All CMEs are accompanied by local brightening in CME source regions. However, according to our definition, 162(82.2\%) CMEs belong to Category A(a), 11(5.6\%) belong to Category A(b), and 24 (12.2\%) belong to Category B. From the above, we know that category $\mathrm{A}(\mathrm{a})$ and $\mathrm{A}(\mathrm{b})$ are related to flares, while $\mathrm{A}(\mathrm{a})$, most of $\mathrm{A}(\mathrm{b})$ and category $\mathrm{B}$ are correlated with filament eruptions. That is to say that there are $87.8 \%$ halo-CMEs are associated with flares, while at least $94.4 \%$ are associated with filament eruptions (with flares or with little brightening). All of these solar activities are accompanied by propagating dimming. Meanwhile, we find that all the CMEs in our sample were accompanied by brightening of CME source regions in the localized areas, although sometimes the intensity increase is so small that we do not regard such brightening as flares.

From these results, we find that halo CMEs and solar surface activity are closely correlated. What is more, more than $90 \%$ events in Category A(a) are identified to be eruptive flares which present two obvious ribbons and post-flare loops, while $82.2 \%$ of the solar disk magnetic activity belong to Category A(a). This is indicative that eruptive flares are tightly linked to CMEs, which clarify and confirm many previous results about CMEs and their associated solar activity.

It was found that eruptive flares were often preceded by filament activation (Svestka \& Cliver 1991). Unfortunately, there is no clear definition of eruptive flares. Zhang et al (2001b) studied a clear instance of a halo CME initiation which was associated with an eruptive flare. They pointed out that the filament first erupted from the region where the magnetic tension and pressure were weaker. The eruptive filament tore open the pre-existing closed magnetic loop (Kopp \& Pneuman 1976). In addition, magnetic reconnection in the upper corona took place, and plasma inside the reconnection region was strongly heated. Hot plasma brightened the magnetic loop region by thermal conduction, shocks, and/or non-thermal particles generated in the reconnection process, and thus produced tworibbon flares with post-flare loop activity (Choudhary \& Gary 1999). According to these characteristics, we suggest to define eruptive flares as a kind of solar disk magnetic activity associated with filament eruptions which lead to flares with the characteristics of two-ribbons and post-flare loops. Eruptive flares are regarded as events of both flare and filament eruptions.

With regard to the locations of CME source regions, the statistics shows that the majority of CMEs initiate from active regions and for about half of the CMEs, the source regions and associated surface activity lie in offsets of CME spans. The detailed statistics are shown in Table 3.

In terms of flares and filament eruptions, this study has revealed a rather close correlation between CMEs and surface activity. Although 197 CMEs are selected as frontside ones in a total of 519 events, the other 322 CMEs are not all from the backside. There are two reasons for us to exclude these 322 CMEs. Among them, Some CMEs with high cadence EIT data are excluded because no associated activity was observed on the visible disk. Those CMEs may not include frontside ones, but the others with low cadence or no EIT data that are excluded may include some frontside ones. However, even though we do not include such uncertain frontside events, we can still get a slight higher correlation rate compared to previous studies. In all CMEs, only two conditions exist, frontside and backside, thus half of the CME events should belong to the 
Table 3. Statistic results about the locations of CME source regions.

\begin{tabular}{lll}
\hline \hline Category & Number & Percentage (\%) \\
\hline Inside activity regions & 155 & 78.7 \\
Outside active regions & 42 & 21.3 \\
Symmetric to CMEs & 96 & 48.7 \\
Asymmetric to CMEs & 101 & 51.3 \\
\hline
\end{tabular}

frontside, that is to say, $259 \mathrm{CMEs}$ are possible. We find $76 \%$ (197/259) CMEs to be associated with surface activity, which is higher than that of most previous statistical results.

Although we reveal a very close correlation between CMEs and surface activity in our sample, we cannot assume the relation between CMEs and associated solar magnetic activity is "cause and effect". In our study, if we ignored the errors in determining the CME initial time which, in fact, could be 2030 min by the low cadence of LASCO observations, among 141 CMEs associated with GOES X-ray flares, 83 CME initiations are seen to precede flare onset, while the other 58 are the opposite. Filament eruptions often precede the initiation of the associated CMEs. The SMM CME observations indicated that the estimated departure time of flare-associated CMEs typically preceded the flare onsets (Webb 2000). Harrison et al. (1990) summarized that CME onsets preceded any related flare activity and that the associated flare commonly lay to one side of the CME span. The CME launch appeared to be associated with minor X-ray (flare precursor) activity. Our data do not allow us to see the soft X-ray precursor. Either on the temporal relationship or on the spatial correlation between CMEs and surface activity, our statistics indicate two possibilities, i.e., the surface activity could precede or follow the associated CME and it could be symmetric or not to the CME. Roughly, each situation shares an approximate $50 \%$ possibility.

\section{Concluding remarks}

We have selected 197 of 519 halo CMEs during the period from 1997 March to 2001 whose source regions are well observed on the solar disk in EIT $195 \AA$ images. We find that 173(88\%) halo CMEs are associated with flares, while more than $187(94 \%)$ are related to filament eruptions. Among these halo CMEs, 78\% are definitively found to correlate with eruptive flares. There are $79 \%$ halo CMEs whose source regions are inside active regions and $21 \%$ are outside active regions. About $50 \%$ of the $\mathrm{CME}$ source regions are symmetric with corresponding CMEs, and about $50 \%$ are asymmetric.

In this study, high quality full disk EUV data are used to identify the CME source regions and the surface activity, which are different from previous studies (Munro et al. 1979; Webb \& Hundhausen 1987; Cyr \& Webb 1991). We concentrate on the frontside halo CMEs and try to find their on-disk counterparts in EIT and other observations, thus to explore the correlation between CMEs and solar surface activity.

We have found that: (1) All 197 studied CMEs are associated with either flares or filament eruptions or both; (2) All the CMEs are accompanied by localized brightening of different extents in their source regions, although sometimes the brightening is too weak to be called flares. Otherwise, all the CMEs are associated with flares; (3) About $78 \%$ of the halo CMEs are definitively associated with eruptive flares. (4) Almost four fifths of the halo CMEs have their source regions inside active regions; (5) Asymmetric events are almost equal to symmetric ones. (6) Among these halo CMEs associated with GOES X-ray record flares, there are $59 \%$ of the CMEs that precede their corresponding flare onsets, while $41 \%$ follow their disk counterparts;

It should be mentioned that several key factors may bring uncertainties to the statistics. The low cadence of LASCO and EIT observations would not allow us to accurately determine the time of flare or CME onset. Possible errors in classifying the associated surface activity could not be excluded. Moreover, lack of coronal observations in the range of 1.0 $2.2 R_{\odot}$ severely constrained us from directly imaging the initiation of CMEs. The identity of filament eruptions for many events in our sample suffers from some ambiguities for the cases when the $\mathrm{H} \alpha$ data were not available. However, since we only choose the frontside halo CMEs that usually are relatively big events, the identity of associated surface activity is easier and will have less ambiguity. On the other hand, our sample covers data almost for 5 years and approximate 200 CMEs are selected for the study. This may make the results statistically meaningful. In addition, in our approach, we have considered the errors in our data base, allow for this in our statistical analysis. Therefore the statistics may shed some light on the physical links between large-scale CMEs and small-scale surface activity.

The current statistics based on a sample of 197 frontside halo CMEs reveals a close correlation of CMEs with surface activity. There are two possible reasons for this. One is that only halo CMEs are selected as the samples whose corresponding surface activity may be comparatively more obviously observed; The other is the rather high-cadence EIT data, provided by the SOHO team, which are made into EIT \& EIT RD movies to show surface activity clearly. Thus the close correlation indicates an intrinsic link between large-scale solar activity, seen as CME, and active-region-scale activity, manifested as flares and filament eruptions. We further confirm the close connection between CMEs and eruptive solar flares. This seems to support a unified view of solar magnetic activity (Slibata 2001; Svestka 2001). We would speculate that some large-scale magnetic structures which form CMEs may have, at least, a part lying in a magnetic complex region, i.e. the source region. The surface activity in the source region may represent a part of the whole process which destabilizes the large-scale structure 
and results in the rather global magnetic eruption in a CME. Therefore, all the CMEs would be accompanied by some activity in the source regions, in the forms of either flares, or filament eruptions, or both. However, the rather small-scale activity, say in an active region, may not necessarily be able to cause the destabilization of large-scale magnetic structure. This is why many flares are not seen to associate with CMEs.

Further efforts are being undertaken to understand the large-scale magnetic structures of the sample CMEs and their correlation with the evolution of the CME source regions.

Acknowledgements. The work is supported by the National Natural Science Foundation of China (G19973009) and the National Key Basic Research Science Foundation (G2000078404). The authors are indebted to the anonymous referee whose suggestions and comments led to improvement of the manuscript. We are grateful to all members of the SOHO EIT, LASCO and MDI teams, as well as BBSO team, Yohkoh team, HSTRC team, HAFB team and for HSOS staff providing the wonderful data. SOHO is a project of international cooperation between the European Space Agency and NASA. We also thank YuMing, Wang and Seiji, Yashiro and K. P. Dere for giving us guidence \& valuable suggestions during this study.

\section{References}

Biesecker, D. A., Thompsom, B. J. 2000, J. Atmospher. and Sol.-Terrestri. Phys., 62, 1449

Bond, H. E., Mullan, D. J., O'Brien, M. S., \& Sion, E. M. 2001, ApJ, 560,919

Choudhary, D. P., \& Gary, G. A. 1999, Sol. Phys., 188, 345

Feynman, J., \& Martin, S. F. 1995, J. Geophys. Res., 100, 3355

Gilbert, H. R., Holzer, T. E., Burkepile, J. T., \& Hundhausen, A. J. 2000, ApJ, 537, 503

Gopalswamy, N., Kaiser, M. L., MacDowell, R. J., et al. 1999, Solar Wind Nine, 471, 641

Gosling, J. T. 1993, J. Geophys. Res., 98, 18, 937-18, 949

Harrison, R. A., Hildner, E., Hundhausen, A. J., Sime, D. G., \& Simnett, G. M. 1990, J. Geophys. Res., 95, 917
Harrison, R. A., \& Lyons, M. 2000, A\&A, 358, 1097

Howard, R. A., Michels, D. J., Sheeley, Jr. N. R., \& Koomen, M. J. 1982, ApJ, 263, 101

Howard, R. A., Bruecker, G. E., St. Cyr, O. C., et al. 1997, GM 99., American Geophys. Union, 17

Hudson, H. S., Lemen, J. R., St. Cyr, O. C., Sterling, A. C., \& Webb, D. F. 1998, Geophys. Res. Lett., 25, 2481

Hudson, H., Haisch, B., \& Strong, K. T. 1995, J. Geophys. Res., 100, 3473

Kopp, R. A., \& Pneuman, G. W. 1976, Sol. Phys., 50, 85

Munro, R. H., Gosling, J. T., Hildner, E., et al. 1979, Sol. Phys., 61, 201

Plunkett, S. P., Thompson, B. J., Howard, R. A., et al. 1998, Geophys. Res. Lett., 25, 2477

Shibata, K., \& Tanuma, S. 2001, Earth Planets Space, 53(6), 473

Sterling, A. C., Hudson, H. S., Thompson, B. J., \& Zarro, D. M. 2000, ApJ, 532, 628

St. Cyr, O. C., \& Webb, D. F. 1991, Sol. Phys., 136, 379

St. Cyr, O. C., Howard, R. A., Simnett, G. M., et al. 1997, ESA SP415, Noordwijk, The Netherlands, ESTECS, 103

St. Cyr, O. C., Howard, R. A., Sheeley, N. R. Jr., et al. 2000, J. Geophys. Res., 105(A8), 18169

Subramanian, P., \& Dere, K. P. 2001, ApJ, 561, 372

Svestka, Z., Cliver, E. W. 1991, LNP, 399, 1

Svestka, Z. 2001, Space Sci. Rev., 95(1/2), 135

Thompson, B. J., Plunkett, S. P., Gurman, J. B., et al. 1998, Geophys. Res. Lett., 25, 2465

Wang, Y. M., Ye, P. Z., Wang, S., Zhou, G. P., \& Wang, J. X. 2002, J. Geophys. Res., in press

Webb, D. F. 2000, J. Atmospher. and Sol.-Terrestri. Phys., 62, 1415

Webb, D. F., \& Hundhausen, A. J. 1987, Sol. Phys., 108, 383

Webb, D. F., Cliver, E. W., Crooker, N. U., St. Cyr, O. C., \& Thompson, B. J. 2000, J. Geophys. Res., 105, 7491

Yashiro, S., Gopalswamy, N., Michalek, G., et al. 2002, J. Geophys. Res., submitted

Zhang, J., Dere, K. P., Howard, R. A., Kundu, M. R., \& White, S. M. 2001a, ApJ, 559, 452

Zhang, J., Wang, J. X., \& Nitta, N. 2001b, Chinese J. Astron. Astrophys., 1(1), 85 